\title{
A flexible and interactive software package for a laboratory in cognition and perception
}

\author{
SARAH RANSDELL \\ University of Maine, Orono, Maine \\ and \\ C. MICHAEL LEVY \\ University of Florida, Gainesville, Florida
}

\begin{abstract}
This paper introduces the second edition of a software package for use in teaching cognitive psychology and research methods. Detailed are the distinctive features of the edition and how this courseware can be used effectively. Fifteen content programs were developed to provide multiple levels of use, ranging from simple demonstrations to sophisticated templates for designing complex independent research projects. Relative to its predecessor, data handling is greatly enhanced, the overall power of the programs to respond to a wider range of responses is increased, and the breadth of the content is expanded significantly.
\end{abstract}

The purpose of this paper is to introduce the second edition of Laboratory in Cognition and Perception, a software package for teaching cognitive psychology and research methods, primarily at the undergraduate level (Levy \& Ransdell, 1989). The features that distinguish this edition from its predecessor (Fischler, Griggs, Warner, Sherman, \& Levy, 1979) are detailed, together with the objectives, pedagogical innovations, and content of the new package. Differences between this package and several others are then outlined, and information about availability and hardware requirements is given.

\section{Overview}

The second edition of this package contains a student workbook and compiled BASIC code distributed on several diskettes. The workbook serves as both a user guide and a reference manual for the software. It details the objectives of the package: to increase the student's understanding of classic research paradigms and findings in cognitive psychology and to provide direct experience with research design and methodology. The 15 substantive chapters of the workbook relate to the 15 individual experiments embodied in the software. ${ }^{1}$ Each chapter introduces a particular paradigm and puts it into a historical, theoretical, or methodological context before laying out the procedural details for the student to follow in using the software. It also guides the student through possible data analyses and attempts to channel the student's thinking about the experiment with questions that might be addressed in a discussion of the results. Finally, the workbook describes how the standard experiments (having only a limited range of possible variations) can be extended to help the student conduct an independent piece of

Correspondence may be sent to Sarah Ransdell, 301 Little Hall, Department of Psychology, University of Maine, Orono, ME 04469. research (using a greatly expanded set of experimental variations).

\section{Objectives}

Throughout this package are features that emphasize the interactive nature of psychological experimentation. When using these materials, students are continuously making decisions comparable to those made by professional researchers. They also come to understand the specific nature of the task as seen by the respondent. Castellan (1988) has stressed the need for tool-based courseware that can provide flexibility and an interactive quality that promotes learning through the techniques of on-line experimentation. Butler (1988) called for more efficient software, particularly experiment simulators that provide both efficiency and flexibility. The present materials address these concerns by increasing the ease with which students can manipulate and create experiments of their own design.

\section{Modes of Use}

One limitation of the first edition of the software was that modifying the code to enable experimental variations that had not been built in was extremely difficult for many instructors and students. A second limitation was that no provision was made for either an instructor or student to preview an experiment: once a program was started, it was necessary to run it completely or to reboot the computer. The present materials overcome these limitations by providing three modes of use for the programs.

The Demonstration mode enables anyone to preview an experiment; sample instructions are given, followed by a small number of trials, and prototypic results are displayed. The primary intent was to allow the student to view the classic design and become familiar with the independent and dependent variables and their potential 
relationships, but instructors and assistants find this mode helpful in refreshing them on procedural details that might need to be elaborated or clarified for the student audience.

The Laboratory mode presents the experiment as detailed in the workbook. It requires the student's input for levels of the independent variable, type of dependent measure, particular keys selected for making responses, and so forth, and permits actual data collection from each administration of the experiment. Once students are familiar with the impact of the basic manipulations, they are ready to make more sophisticated and creative design choices.

In the most flexible mode, Advanced Projects, students can produce designs to test unique hypotheses about a set of psychological concepts. The following example may help to illustrate the nature of this feature.

One of the possible experiments deals with the topic of reading. This exercise focuses on the word-by-word technique (Aaronson \& Ferres, 1984) in which, as the subject presses a key, the word on the screen is extinguished and is immediately replaced by the next word in a sentence. The computer records the time that each word is exposed and, hence, measures reading time. In the Laboratory mode, four independent variables are manipulated: (1) type of test (recall, comprehension, or both), (2) practice (examine results from the first or last half of the experiment), (3) voice (i.e., whether the sentence is in active or passive voice), and (4) category of the word (noun, adjective, etc.). The dependent variables are reading time for each word and the percentage of correctly recalled words (if a recall measure is included).

In the Advanced Projects mode, students can manipulate the following variables: (1) possible stimuli (that is, use stimuli already on disk or those created by the student or provided by the instructor); (2) reading strategy (whether the subject is told which of the two types of tests will be given preceding the trials or after); (3) whether response time is measured for each word, for each sentence, or for both; and (4) the instructions given to each subject (e.g., to view the effects of speed vs. accuracy). Manipulations 1 and 4 in particular allow divergent thinking to enter into design decisions. In a typical application of this mode, students first write a brief research proposal outlining a new design with a corresponding rationale.

When the instructor supplies a password to the main menu of the programs, a hidden screen appears that enables the access modes to be set independently for each experiment.

\section{Data Handling}

The data collected from a student's run through the Laboratory mode of an experiment are stored in two files: (1) an ASCII sequential file that can be downloaded to external statistical packages or spreadsheets and (2) a file containing summarized and formatted results that can be printed immediately or later. Data from Advanced Projects are stored in sequential data files. Maps of these files, identifying the contents of each cell, are provided in the appendices to the workbook.

\section{Addition of Contemporary Topics}

Cognitive psychology is such a rapidly expanding field that new paradigms and methods must be introduced into a laboratory class with some regularity. Accordingly, seven completely new topics were added to the package: spatial cognition, activation of memory, implicit memory, lateralization of language, reading, acquisition of natural concepts, and problem solving.

\section{Increased Program Power}

The 1979 edition of the lab package was originally designed to run on a minicomputer whose multi-user BASIC divided memory into $8 \mathrm{~K}$ partitions for each workstation, severely limiting the overall capabilities and performance of the software. Because the intended environment for the new materials is one based on single-user microcomputer-based workstations, where memory constraints are no longer a concern, the new code can provide detailed instructions and help messages, elaborate error checking and response verification, and enhanced flexibility (e.g., in the response options).

The code was written in BASIC to facilitate distribution and the task of migrating the code to different processors. Assembly-language routines were incorporated for control of stimulus timing and measurement of response latencies; these routines self-adjust to the speed of the system running the programs. The runtime versions have been compiled.

\section{A Comparison to Other Current Software}

The package described here clearly takes a different approach from that of Gregory and Poffel's (1989) START programs, which are excellent tools to help a student to set up an experiment; the researcher using START must engage in considerably more preparation for a project than do students using our materials. Keenan and Keller's (1987) Computer Lab in Memory and Cognition shares many features with our materials (especially the attention each gives to guiding the student's analyses and understanding of cognitive processes), but differs in that its focus-and therefore its audience-is much narrower. Schneider's (1988) Micro Experimental Laboratory is a powerful ensemble of tools that can be used in teaching research methods in cognitive psychology; however, it is aimed at a much more sophisticated group of users than are any of the other programs listed.

\section{Hardware Requirements and Availability}

The software package will run on IBM PC, XT, AT, and PS/2-compatible machines with at least $256 \mathrm{~K}$ memory and one floppy disk drive (two drives or a hard drive are recommended). Several programs require graphics and, thus, CGA, EGA, VGA, or Hercules-compatible monochrome adapters. A printer is not required, but is highly 
desirable. Versions for the Apple II-series and Macintosh computers are forthcoming.

The package is published by CONDUIT, University of Iowa, Oakdale Campus, Iowa City, IA 52242, which can provide single, multiple, network, and site licenses. Desk copies of the workbooks are provided with the software licenses, and student copies are customarily ordered for sale through college bookstores.

\section{REFERENCES}

Aaronson, D., \& Ferres, S. (1984). The word-by-word reading paradigm: An experimental and theoretical approach. In D. Kieras and $\mathrm{M}$. A. Just (Eds.), New methods in reading comprehension (pp. 31-68). Hillsdale, NJ: Erlbaum.

BUTLER, D. L. (1988). Selection of software in the instructional laboratory. Behavior Research Methods, Instruments, \& Computers, 20, 175-177.

Castellan, N. J., JR. (1988). Comments on applications of microcomputers in teaching. Behavior Research Methods, Instruments, \& Computers, 20, 193-196.
Fischler, I., Griggs, R. A., Warner, S. A., Sherman, M. E., \& LEVY, C. M. (1979). Laboratory in cognition and perception. Iowa City, IA: CONDUIT.

GREGORY, R. J., \& PoFFel, S. A. (1989). START: Stimulus and response tools for experiments in memory, learning, cognition and perception. Iowa City, IA: CONDUIT.

Keenan, J. M., \& Keller, R. A. (1987). Computer lab in memory and cognition. Iowa City, IA: CONDUIT.

LEVY, C. M., \& RANSDELL, S. E. (1989). Laboratory in cognition and perception (2nd ed.). Iowa City, IA: CONDUIT.

SCHNEIDER, W. (1988). Micro Experimental Laboratory: An integrated system for IBM PC compatibles. Behavior Research Methods, Instruments, \& Computers, 20, 206-217.

\section{NOTE}

1. These experiments are named Classical Psychophysics, Signal Detection, Sensory Memory, Feature Detection, Spatial Cognition, Retrieval from Working Memory, Activation of Long-Term Memory, Memory without Awareness, Visual and Semantic Comparisons, Lateralization of Language, Reading, Formal Concepts, Natural Concepts, Reasoning from Prose, and Problem Solving. 